\title{
A Combined Reliability Model of VSC-HVDC Connected Offshore Wind Farms Considering Wind Speed Correlation
}

\author{
Guo, Yifei; Gao, Houlei; Wu, Qiuwei
}

Published in:

IEEE Transactions on Sustainable Energy

Link to article, DOI:

10.1109/TSTE.2017.2698442

Publication date:

2017

Document Version

Peer reviewed version

Link back to DTU Orbit

Citation (APA):

Guo, Y., Gao, H., \& Wu, Q. (2017). A Combined Reliability Model of VSC-HVDC Connected Offshore Wind Farms Considering Wind Speed Correlation. IEEE Transactions on Sustainable Energy, 8(4), [7912299]. https://doi.org/10.1109/TSTE.2017.2698442

\section{General rights}

Copyright and moral rights for the publications made accessible in the public portal are retained by the authors and/or other copyright owners and it is a condition of accessing publications that users recognise and abide by the legal requirements associated with these rights.

- Users may download and print one copy of any publication from the public portal for the purpose of private study or research.

- You may not further distribute the material or use it for any profit-making activity or commercial gain

- You may freely distribute the URL identifying the publication in the public portal 


\title{
A Combined Reliability Model of VSC-HVDC Connected Offshore Wind Farms Considering Wind Speed Correlation
}

\author{
Yifei Guo, Houlei Gao, Member, IEEE, and Qiuwei Wu, Senior Member, IEEE
}

\begin{abstract}
This paper proposes a combined reliability model of voltage source converter-based high voltage direct current (VSCHVDC) connected offshore wind farms (WFs) using the frequency and duration technique. Firstly, a two-dimensional multi-state WF model is developed considering wind speed variations and WTGs outage. The wind speed correlation between different WFs is included in the two-dimensional multistate WF model by using an improved k-means clustering method. Then, the entire system with two WFs and a threeterminal VSC-HVDC system is modeled as a multi-state generation unit. The proposed model is applied to the Roy Billinton test system (RBTS) for adequacy studies. Both the probability and frequency indices are calculated. The effectiveness and accuracy of the combined model is validated by comparing results with the sequential Monte Carlo simulation (MCS) method. The effects of the outage of VSC-HVDC system and wind speed correlation on the system reliability were analyzed. Sensitivity analyses were conducted to investigate the impact of repair time of the offshore VSC-HVDC system on system reliability.
\end{abstract}

Index Terms-- Adequacy assessment, analytical reliability model, clustering technique, frequency and duration technique, VSC-HVDC, wind power.

\section{NOMENCLATURE}

ACF AC filter.

Brk Breaker.

Cap DC capacitor.

CR Converter reactor.

DCF DC filter.

DCTL DC transmission line.

DFIG Doubly fed induction generator.

GSVSC Grid side voltage source converter.

PCS Protection and control system.

Trn Transformer.

Valves Vlv.

This work was supported by the National Key Research and Development Program of China under Grant 2016YFB0900603.

Y. Guo and H. Gao are with the Key Laboratory of Power System Intelligent Dispatch and Control of Ministry of Education, Shandong University, Jinan 250061, China (e-mail: yfguo_sdu@163.com; houleig@sdu.edu.cn).

Q. Wu is with the Center for Electric and Energy, Department of Electrical Engineering, Technical University of Denmark, Lyngby 2800, Denmark, the Sino-Danish Center for Education and Research, Aarhus 8000, Denmark, and also with the School of Electrical Engineering, Shandong University, Jinan 250061, China (e-mail: qw@elektro.dtu.dk). $\begin{array}{ll}\text { VSC } & \text { Voltage source converter. } \\ \text { WF } & \text { Wind farm. } \\ \text { WFVSC } & \text { Wind farm side voltage source converter. } \\ \text { WTG } & \text { Wind turbine generator. }\end{array}$

\section{INTRODUCTION}

TIND power has been developing very fast over the world due to the environmental concern and energy supply security issue. Large-scale wind power projects have been rapidly developed in Europe. The European Wind Energy Association (EWEA) states that the installed capacity of wind power projects could expand to $320 \mathrm{GW}$ by 2030 [1].

The integration of large-scale wind farms (WFs) creates a number of technical and economic challenges for developers and operators due to the variability and uncertainty of wind [2]. The outputs of wind turbine generators (WTGs) are determined by wind condition and availability of WTGs. The intermittent characteristics of wind energy and random nature of failures of WTGs make the generation output of WFs stochastic and totally different from that of conventional generating units. Consequently, it complicates reliability assessment and management [3].

Reliability assessment of power systems incorporating wind energy has been widely studied. The assessment methods can be generally classified into two types: 1) analytical methods [3]-[7]; and 2) Monte Carlo simulation (MCS) methods [8]-[12]. In [3], the time-varying reliability models of WFs, conventional generating units, and rapid start-up generating units were developed and represented as the corresponding universal generating functions. In [4], a multistate model of a WF based on the apportioning method was developed. The simulation results show that a five-state model can be used to provide a reasonable assessment of the power system adequacy studies using analytical methods. In [5]-[6], the analytical models of WFs were developed considering the stochastic characteristics of wind speed and outage of WTGs. A comprehensive analytical method for reliability modeling of doubly fed induction generator (DFIG)-based WFs was proposed in [7]. In [8]-[10], the effects of large-scale WFs on power system adequacy assessment were investigated using the MCS method. In [11], a two-dimensional wind speed statistical model was developed and used in the reliability assessment of microgrids based on the sequential MCS. A time-dependent model of WFs was studied and applied to 
reliability assessment using the state-sampling MCS method in [12]. The proposed method shows better performance than the sequential MCS on the computation efficiency.

Previous studies have shown that the sequential MCS method can model the chronological characteristics of wind speed as well as load. Therefore, it can precisely reflect the impacts of wind power on power systems. However, the MCS method often requires longer computational time. In contrast, the analytical method is more efficient for reliability assessment. In the analytical method, WFs can be modeled as multi-state conventional units.

Wind speed correlation has a significant impact on system reliability. In [8]-[10], wind regimes were modeled using the autoregressive moving average (ARMA) time series model. The wind speed correlation level between different wind locations can be adjusted by selecting the random number seeds for a random number generation process. In [13], the Copula theory was used to generate correlated wind speed time series and then the multistate model of WFs was developed based on a linear apportioning method.

A considerable number of large-scale WFs are planned offshore. Among the available technologies for the connection of offshore WFs, the voltage source converter-based high voltage direct current (VSC-HVDC) transmission system has become an attractive option for connecting distant offshore WFs due to its flexible active and reactive power control, feasibility of multi-terminal dc or meshed grids and inherent black start capability [14], [15].

The reliability assessment of HVDC systems is important for the growing hybrid AC/DC power systems. A number of studies have been done for the reliability assessment of HVDC systems [16]-[18]. Mostly, these studies focus on the conventional line commuted converter-based HVDC (LCCHVDC), while few studies have been done for the reliability assessment of power systems with VSC-HVDC systems. As mentioned above, due to its economic and technical advantages, VSC-HVDC will play a significant role in the development of offshore wind power. The failures of VSCHVDC systems may cause a complete outage of offshore WFs and consequently zero output. Therefore, the VSC-HVDC system shall be modeled together with WFs in the reliability assessment.

To investigate the reliability of power systems with offshore wind power, a combined analytical reliability model of VSC-HVDC connected offshore WFs is proposed in this paper. Firstly, a two-dimensional multi-state WF model which takes into account both the wind speed variations and WTGs outage is developed. The wind speed correlation between WFs is considered in this model by using an improved k-means clustering method. Then, the reliability model of the VSCHVDC system is developed and the whole system is modeled as a multistate conventional generation unit by combining the reliability models of the WFs and VSC-HVDC system. The proposed model is applied to the Roy Billinton test system (RBTS) for adequacy studies. Both probability and frequency reliability indices are calculated. The effectiveness of the combined model is demonstrated by comparing results with the sequential MCS method. The effects of wind speed correlation and the outage of the VSC-HVDC system are analyzed. Besides, sensitivity analyses are conducted to investigate the effect of repair time of the offshore VSCHVDC system on system reliability.

The rest of this paper is organized as follows. In Section II, the frequency and duration technique is briefly introduced. In Section III, the two-dimensional multi-state model of WFs is developed. In Section IV, the reliability model of the VSCHVDC system is developed and then combined with the WF model. The adequacy assessment method using the proposed analytical combined model is presented in Section V. In Section VI, the proposed model is applied to the RBTS for adequacy studies, followed by conclusions.

\section{FREQUENCY AND DURATION TECHNIQUE}

The Markov approach can be used for systems whose behavior can be described by a probability distribution with a constant hazard rate. Representing wind power by a stationary Markov process implies that the residence time of states follows an exponential distribution. In fact, wind power time series are non-stationary due to seasonal variations of wind. However, this effect can be neglected if the amount of data is large enough such as one year data or more. And it will not be necessary that wind power exactly follows this distribution, if only long-term average values are required [5]. The limiting state probabilities and frequencies of a process having nonexponential distributions are identical to those evaluated under the assumption that the states are exponentially distributed [19]. Thus, a stationary Markov process is used in this paper.

The exponential distribution requires a constant transition rate, which is defined by,

$$
\lambda_{i j}=\frac{N_{i j}}{D_{i}}
$$

where $\lambda_{i j}$ is the transition rate from state $i$ to state $j, N_{i j}$ is the number of transitions from state $i$ and state $j$, and $D_{i}$ is the duration of state $i$ during the whole observed period.

The probability of occurrence of state $i$ is given by,

$$
p_{i}=\frac{D_{i}}{T}
$$

where $T$ is the total observation period.

The frequency of occurrence of state $i, f_{i}$, is then calculated by,

$$
f_{i}=p_{i} \sum_{j \neq i} \lambda_{i j}
$$

\section{RELIABILITY MODEL OF WFS}

In this section, a two-dimensional multi-state model of two WFs with correlated wind speed is developed based on the frequency and duration technique. Fig. 1 shows the modeling procedure for WFs. In Part A, the global fast k-means clustering method used to model the correlation between WFs 
is presented. The power output of a WTG depends on two factors: wind speed and WTG availability. Hence, a twodimensional single-turbine output model and WTG outage model are developed in Part B and Part C, respectively. In the last part, a two-dimensional multi-state WF model is developed using the state-space diagram.

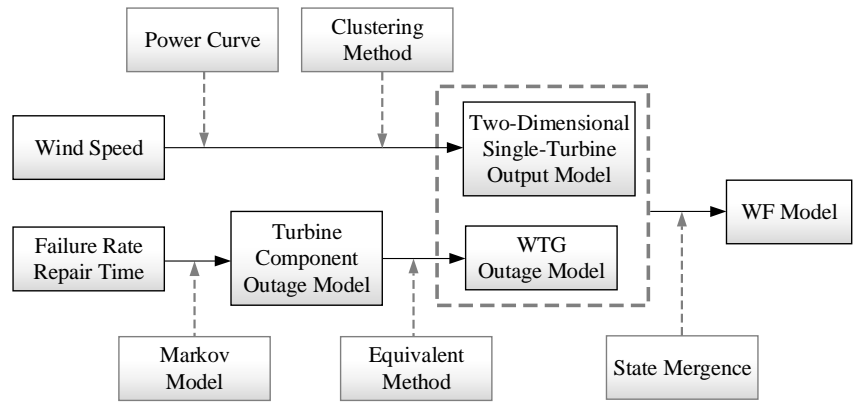

Fig. 1. Modeling procedure for WFs.

\section{A. Global Fast k-Means Clustering Algorithm}

The clustering technique aims to partition a dataset into several well-separated subsets (clusters) based on the similarity criterion. The k-means algorithm is a popular clustering method. However, the conventional k-means algorithm is a local search procedure and its performance heavily depends on the initial conditions. Hence, an improved k-means algorithm, called a global fast k-means clustering algorithm, is used in this paper [20].

Suppose a given dataset $X=\left\{x_{1}, x_{2}, \ldots, x_{N}\right\}, x_{i} \in \Re^{S}$. The clustering method partitions the dataset into $K$ clusters $C_{1}$, $C_{2}, \ldots, C_{K}$ by solving a minimization optimization problem. The objective function is the sum of square Euclidean distances between data point $x_{i}$ and cluster center $v_{k}$ of the dataset $C_{k}$ which contains $x_{i}$. It is defined by,

$$
J\left(C_{1}, C_{2}, \ldots, C_{K}\right)=\sum_{k=1}^{K} \sum_{i=1}^{N} \operatorname{sign}\left(x_{i}, C_{k}\right)\left\|x_{i}-v_{k}\right\|^{2}
$$

where

$$
\operatorname{sign}\left(x_{i}, C_{k}\right)=\left\{\begin{array}{l}
1, \text { if } x_{i} \in C_{k} . \\
0, \text { if } x_{i} \notin C_{k} .
\end{array}\right.
$$

The global fast k-means clustering algorithm proceeds in an incremental way. To solve the problem with $K$ clusters, all intermediate problems with $1,2, \ldots, K-1$ clusters are sequentially solved.

The procedure starts with one cluster $(k=1)$ and the first cluster center corresponds to the centroid of the dataset. Suppose $v_{1}^{(k-1)}, v_{2}^{(k-1)}, \ldots, v_{k-1}^{(k-1)}$ are the cluster centers obtained by solving the $(k-1)$-clustering problem. $d_{1}^{(k-1)}, d_{2}^{(k-1)}, \ldots, d_{N}^{(k-1)}$ are the square distances between $x_{i}$ and the closest cluster center (i.e., the center of the cluster which $x_{i}$ belongs to among these $k-1$ cluster centers). The initial cluster centers for the $k$-clustering problem can be expressed as $\left\{v_{1}^{(k-1)}, v_{2}^{(k-1)}, \ldots, v_{k-1}^{(k-1)}, x_{i}^{*}\right\}$ where the additional initial cluster center $x_{i}^{*}$ is determined by,

$$
i=\arg \max _{w}\left[\sum_{j=1}^{N} \max \left(d_{j}^{(k-1)}-\left\|x_{w}-x_{j}\right\|^{2}, 0\right)\right] .
$$

The detailed procedure of the global fast k-means clustering method is illustrated in Fig. 2.

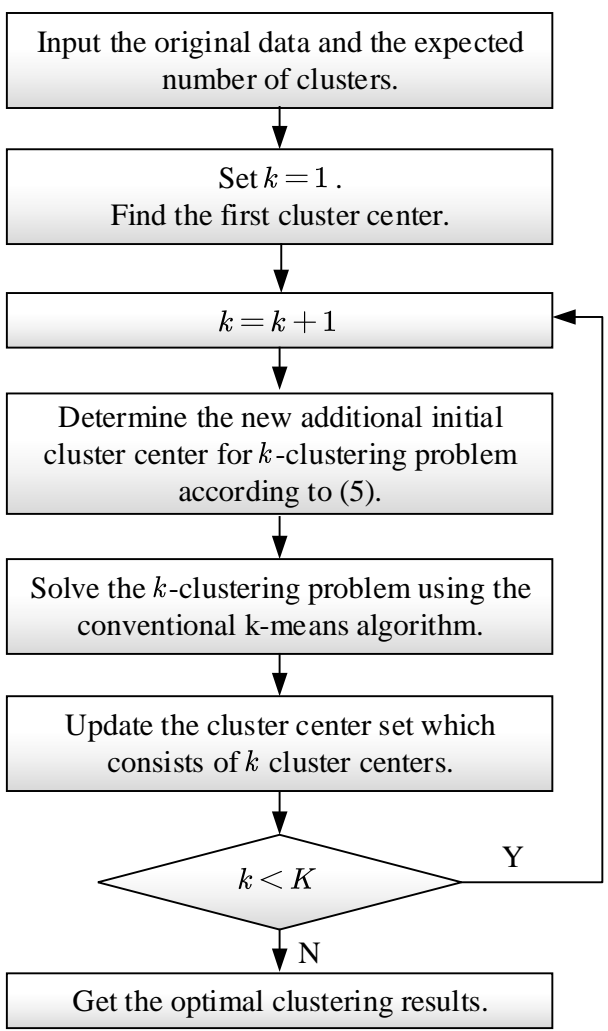

Fig. 2. Flowchart of the global fast k-means clustering algorithm.

\section{B. Single-Turbine Output Model Considering Wind Speed Variations}

If a WTG is operating, its output depends on wind speed as well as the performance characteristics of the generator, which is completely different from the conventional generation units. Therefore, the effect of wind speed variations should be fully considered in the model.

The historical wind speed data of two WFs (WF_1 and WF_2) in eastern China from 2013 to 2015 with 10-min resolutions are used in this work. The statistical data of wind speed are listed in Table I.

TABLE I

WIND SPEED STATISTICAL DATA

\begin{tabular}{c|c|c|c}
\hline \hline WF & Average (m/s) & Standard deviation (m/s) & Correlation \\
\hline \hline WF_1 & 8.4969 & 4.1956 & \multirow{2}{*}{0.7036} \\
\hline WF_2 & 8.2068 & 3.9881 & \\
\hline \hline
\end{tabular}

The hourly wind speed series are obtained by averaging six 10-min values. The power curve of a V90-3.0 MW WTG is used in this study [7]. Fig. 3 shows the two-dimensional 
scatter diagram of the power output of a single WTG in the two WFs with correlated wind speed.

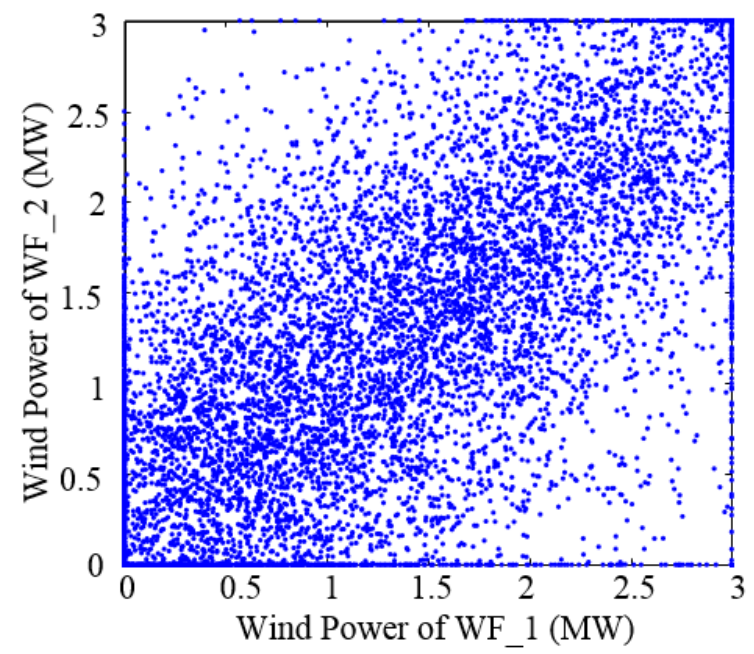

Fig. 3. Scatter diagram of the wind power.

In existing work, the analytical models of a single WF for reliability assessment have been studied [5]-[7] whereas multiple WFs are not mentioned. As shown in Fig. 3, the outputs of two WFs are correlated in a certain degree. Therefore, their correlation shall be modelled. To address the problem, a two-dimensional single-turbine output model is presented as follows.

The Markov chain can model the variations of a stochastic process as transitions between states where each state represents a discrete value. The number of states depends on the accuracy requirements of the model. Too many states increase the computational burden. For a 3-MW WTG, the power output can be divided into 0-, 0.5-, 1-, 1.5-, 2-, 2.5-, 3MW steps [6]. If the same approach is used to the models with two or more dimensions, it will be hard to determine the suitable number and range of steps. Hence, the global fast kmeans clustering algorithm is used to partition states.

Fig. 4 shows the objective function values versus the number of clusters. With the increase of the cluster number, the objective function value decreases. However, the reduction of the objective function value becomes insignificant after selecting 15 or more clusters. Considering the accuracy of the model as well as the computation efficiency, a 20-state model is thus used in this work.

Fig. 5 shows the clustering centers which can be used to represent the power output states. As shown in Fig. 5, the clusters can effectively represent the correlation between two WFs. Fig. 6 shows the state-space diagram of the twodimensional 20-state single-turbine output model. For the sake of legibility, some of the transitions between non-adjacent states are not shown in Fig. 6.

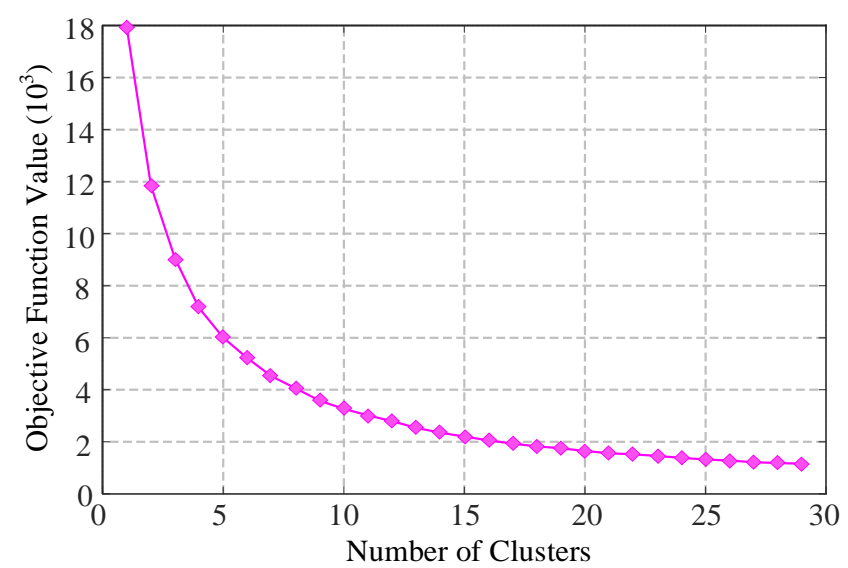

Fig. 4. Objective function values with different number of clusters

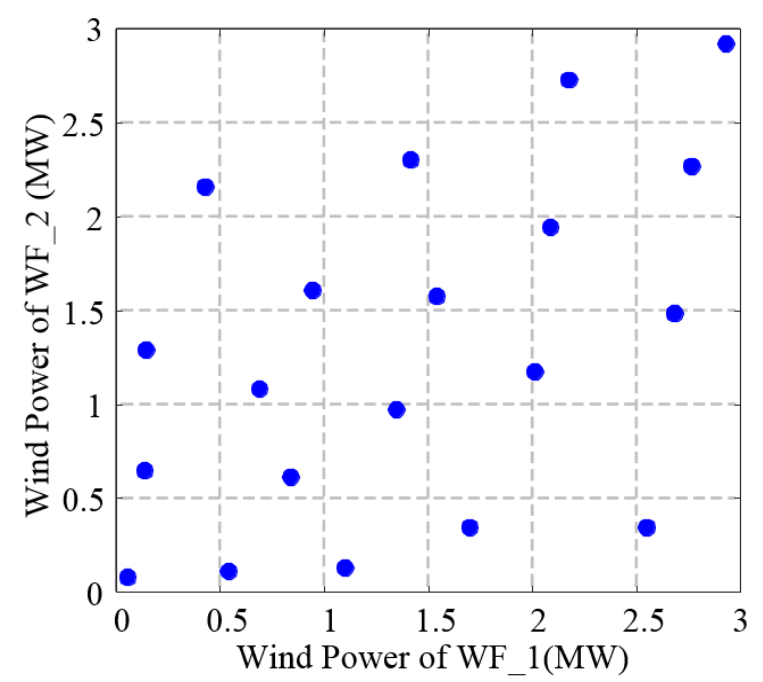

Fig. 5. Cluster centers.

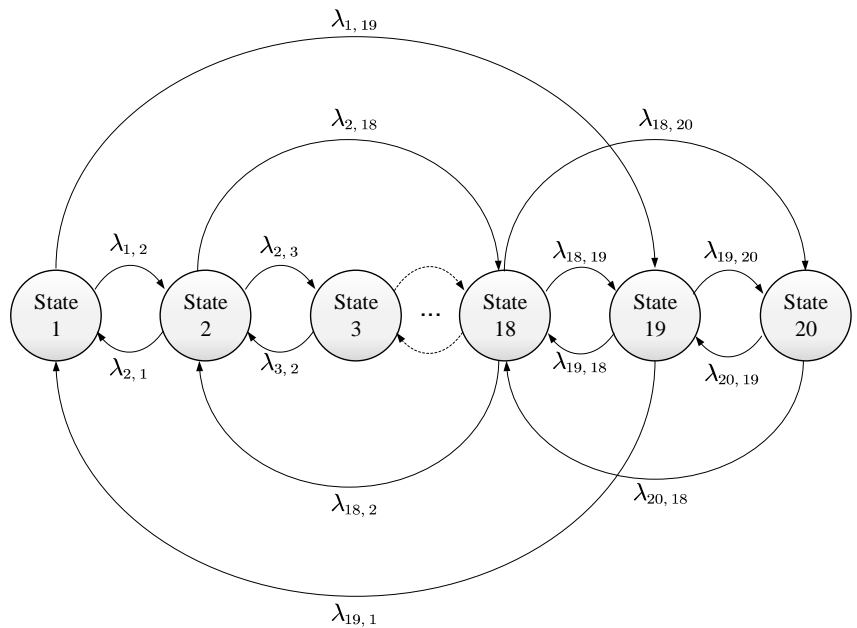

Fig. 6. State-space diagram of the two-dimensional single-turbine output model considering wind speed variations. 


\section{Outage Model of a WTG}

A WTG as a repairable system is comprised of a number of components. Failures of some crucial components will result in a complete outage of the WTG. Fig. 7 shows a simple twostate reliability model for each turbine component, in which the component is either in the up or down state [7]. An equivalent two-state Markov model of a system with $n$ series components can be obtained using the equations as follows [21],

$$
\begin{array}{r}
\lambda_{\mathrm{eq}}=\sum_{i=1}^{n} \lambda_{i} \\
r_{\mathrm{eq}}=\frac{\sum_{i=1}^{n} \lambda_{i} r_{i}}{\sum_{i=1}^{n} \lambda_{i}}
\end{array}
$$

where $\lambda_{i}$ and $r_{i}$ denote the failure rate, and repair time of the component, and $\lambda_{\text {eq }}$ and $r_{\text {eq }}$ are the unit equivalent failure frequency and outage time, respectively.

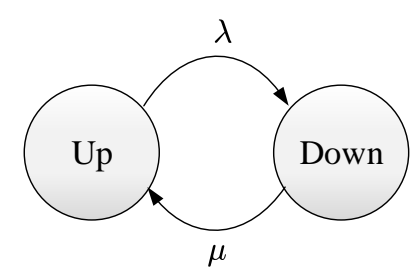

Fig. 7. Two-state Markov model of WTG components.

DFIG-based WTGs are considered in this paper. The WTG components outage statistics are based on a survey of failures in the Swedish wind power projects during 1997-2005 [22], which are listed in the Appendix. The equivalent failure rate and repair time of a DFIG-based WTG are 0.351 occ/yr and $150.9 \mathrm{~h}$, respectively.

\section{Multistate Model of WFs}

The WF model can be developed based on the singleturbine output model and outage model of WTGs. A WF with multiple WTGs is subject to the same wind regime. The reliability model of two WFs can be established by combining the two-dimensional model and operating state model (up or down). Suppose the two WFs are comprised of ten 3-MW WTGs each. The state space diagram of the WFs is shown in Fig. 8. In each state-circle, the number above is the label number of the state, and the numbers at the lower left and right corners are the available number of WTGs in WF_1 and WF_2, respectively. For two $N$-turbine WFs, the total number of states is $(N+1)^{2}$. The probability of a WF with $M$ available WTGs $p(M)$ can be calculated by,

$$
p(M)=\frac{N !}{M !(N-M) !} A^{M} U^{N-M}
$$

where $A$ and $U$ are the availability and unavailability of the WTGs, respectively.

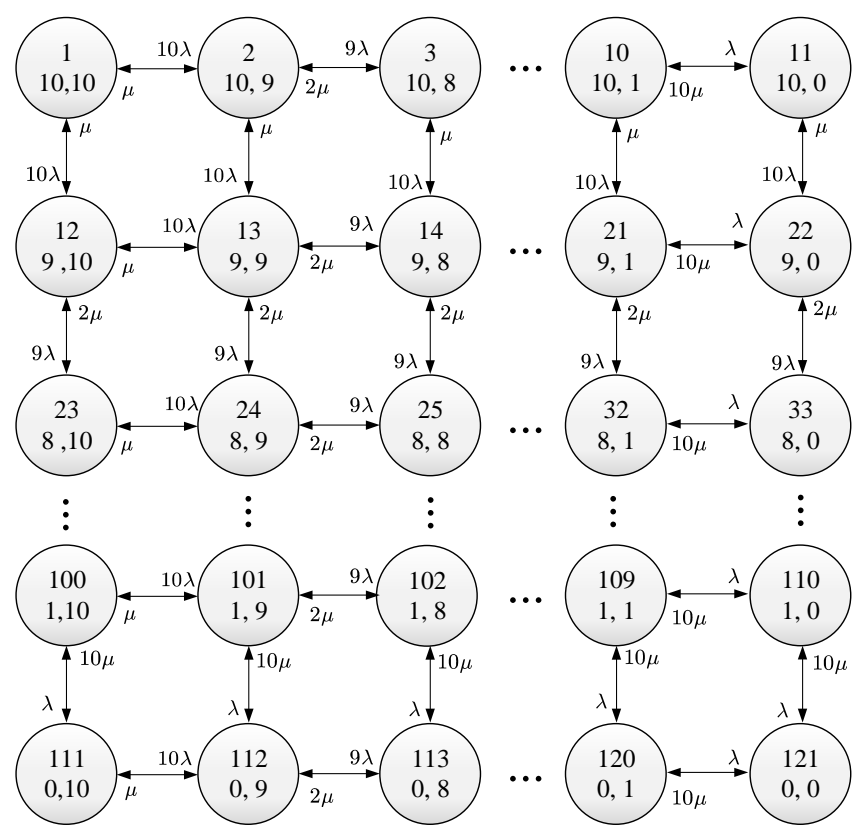

Fig. 8. State-space diagram of two WFs.

For two $N$-turbine WFs and $n$ power output states for a single WTG, the total number of states in the state space diagram is $n(N+1)^{2}$, which is too large for the modeling. In order to reduce the computational burden, those almost identical states can be merged using the clustering technique. The clustering centers represent the new merged states. The probability and frequency parameters of the merged states are obtained using the following equations [21],

$$
\begin{gathered}
C_{1}=C_{2}=\cdots=C_{i}=C_{Z} \\
p_{Z}=\sum_{i \in A_{Z}} p_{i} \\
f_{Z}=\sum_{i \in A_{Z}} f_{i}-\sum_{(i, j) \in A_{Z}} p_{i} \lambda_{i j} \\
\lambda_{Z_{1}, Z_{2}}=\frac{\sum_{i \in A_{Z_{1}}} p_{i} \sum_{j \in A_{Z_{2}}} \lambda_{i j}}{\sum_{i \in A_{Z_{1}}} p_{i}}
\end{gathered}
$$

where subscript $i$ refers to the state resulting in identical power output, $Z$ refers to the new merged state, $C, p, f$ and $\lambda$ are the state capacity, probability, frequency and transition rate with subscript $i$ for identical states and $Z$ for new merged states, respectively, and $A_{Z}$ is the set of states with identical power output.

According to the objective function value of the clustering method, the suitable number of states for the final reliability model of WFs is 20. Table II shows the final multi-state model of WFs. 
TABLE II

Two-Dimensional MUlti-State Model of WFs

\begin{tabular}{c|c|c|c}
\hline \hline State & $\begin{array}{c}\text { Power output } \\
(\mathrm{MW})\end{array}$ & Probability & $\begin{array}{c}\text { Frequency } \\
\text { (occ/hr) }\end{array}$ \\
\hline \hline 1 & $(0,0)$ & 0.094918 & 0.027119 \\
\hline 2 & $(1.1755,3.7402)$ & 0.000124 & $5.741 \mathrm{E}-5$ \\
\hline 3 & $(4.7110,0.9142)$ & 0.063507 & 0.031450 \\
\hline 4 & $(1.1378,7.4600)$ & 0.077513 & 0.035496 \\
\hline 5 & $(9.2170,1.5286)$ & 0.042426 & 0.021080 \\
\hline 6 & $(5.9104,4.8419)$ & 0.055682 & 0.032323 \\
\hline 7 & $(1.3868,12.8529)$ & 0.041742 & 0.019902 \\
\hline 8 & $(5.1853,9.6171)$ & 0.051086 & 0.030158 \\
\hline 9 & $(13.7161,2.1753)$ & 0.002190 & 0.001091 \\
\hline 10 & $(10.4563,8.3937)$ & 0.003787 & 0.002030 \\
\hline 11 & $(18.8025,2.6357)$ & 0.035058 & 0.017243 \\
\hline 12 & $(7.3585,14.5430)$ & 0.049110 & 0.026511 \\
\hline 13 & $(2.6174,20.8472)$ & 0.016901 & 0.009001 \\
\hline 14 & $(15.9494,10.2426)$ & 0.067906 & 0.034041 \\
\hline 15 & $(25.1372,2.3881)$ & 0.015776 & 0.007281 \\
\hline 16 & $(12.1310,17.5056)$ & 0.060142 & 0.030575 \\
\hline 17 & $(24.3619,11.1535)$ & 0.069550 & 0.028250 \\
\hline 18 & $(19.4448,17.4366)$ & 0.058052 & 0.028920 \\
\hline 19 & $(12.2412,25.2757)$ & 0.034705 & 0.017463 \\
\hline 20 & $(25.1083,24.1173)$ & 0.159824 & 0.021534 \\
\hline \hline
\end{tabular}

\section{COMBINED WFs AND VSC-HVDC RELIABILITY MODEL}

A system comprised of two offshore WFs and a typical three-terminal VSC-HVDC system, shown in Fig. 9, is used in this paper to develop the combined model. Each WF is equipped with a WF side VSC (WFVSC) and the two WFs share a common grid side VSC (GSVSC).

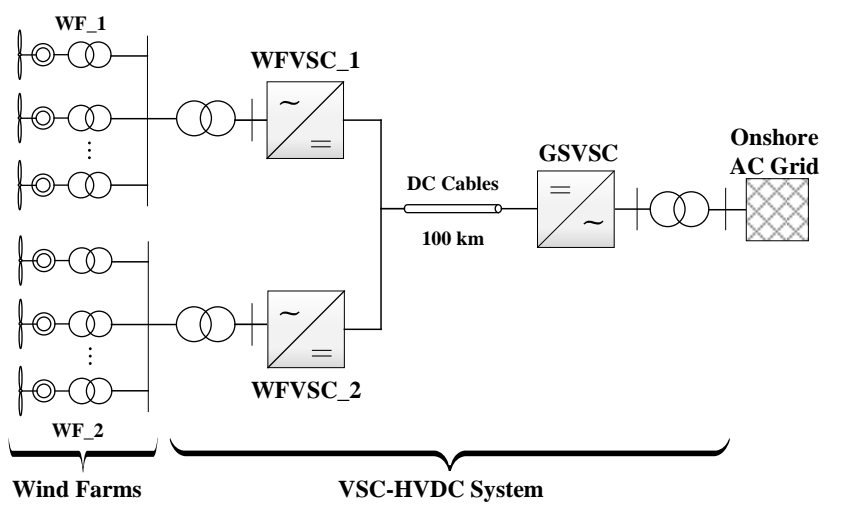

Fig. 9. Structure of the system.

Fig. 10 shows the structure of the combined reliability model of the whole system. The system can be divided into five subsystems. Subsystem 1 is the two offshore WFs. The three-terminal VSC-HVDC system consists of Subsystems 2 to 5. Subsystem 2 and Subsystem 3 represent the two WFVSC stations, and Subsystem 5 represents the common GSVSC station, respectively. Subsystem 4 is the DC transmission line.

\section{A. Reliability Model of VSC-HVDC System}

1) VSC station: A VSC station consists of the following components [23]: 1) ACF; 2) Brk; 3) CR; 4) Trn; 5) Vlv; 6) Cap; 7) DCF; 8) PCS.

Faults may happen on these components of the VSC station.
It is assumed that a fault on any of the components causes a full outage of the VSC station. As such, the series equivalent reliability model of the VSC station can be represented by a typical two-state Markov model using (6.a) and (6.b). The outage data of the VSC-HVDC system are listed in the Appendix [16]-[17], [24]. The equivalent reliability model of the VSC station is listed in Table III.

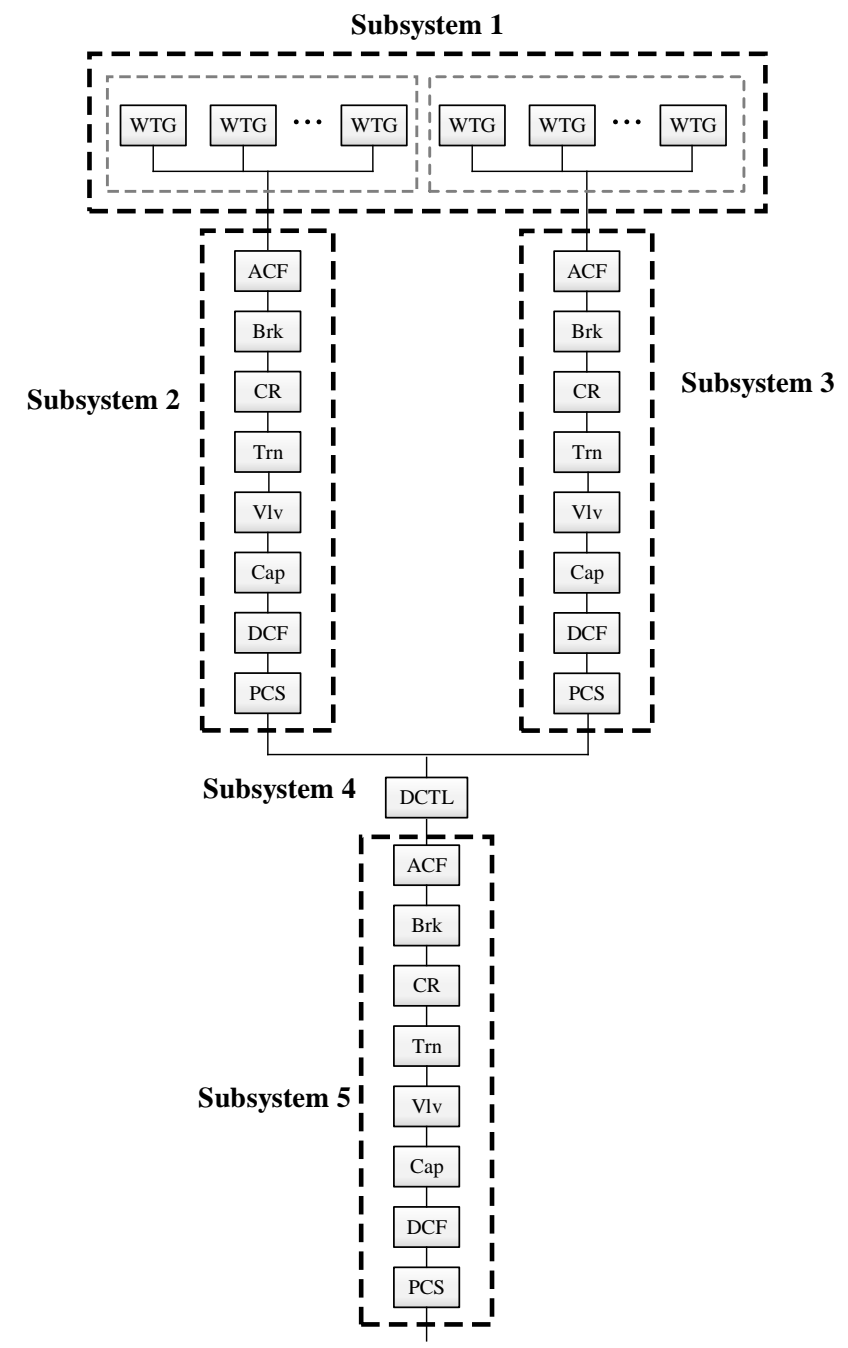

Fig. 10. Structure of the combined reliability model.

TABLE III

RELIABILITY MODELS OF VSC-HVDC SYSTEM

\begin{tabular}{c|c|c|c}
\hline \hline Subsystems & $\begin{array}{c}\text { Capacity in } \\
(\%)\end{array}$ & Probability & $\begin{array}{c}\text { Frequency } \\
\text { (occ/yr) }\end{array}$ \\
\hline \hline \multirow{2}{*}{ VSC station } & 100 & 0.996034 & 4.8619 \\
\cline { 2 - 4 } & 0 & $3.965 \mathrm{E}-3$ & 4.8619 \\
\hline \multirow{2}{*}{ DCTL } & 100 & 0.999997 & $6 \mathrm{E}-3$ \\
\cline { 2 - 4 } & 0 & $3.424 \mathrm{E}-6$ & $6 \mathrm{E}-3$ \\
\hline \hline
\end{tabular}

2) DC transmission line: The system includes two main common parallel DC transmission lines using submarine cables. It is assumed that any failure of the main transmission lines causes a complete outage of the VSC-HVDC system. The failures of medium-voltage collector systems inside WFs 
are not considered in this paper. The length of the main transmission line is assumed to be $100 \mathrm{~km}$. The model of the DCTL is listed in Table III.

\section{B. Combined Reliability Model}

To develop the combined reliability model of the whole system, the WF model and WFVSC model are combined into an equivalent model (EM_1), and the DCTL model and GSVSC model are combined into an equivalent model (EM_2). Then, these two equivalent models are combined into the final combined model. The details are described as follows.

Obviously, the failures of WFVSC_1 and WFVSC_2 influence the power output of WF_1 and WF_2. The statespace diagram of EM_1 for only one power output state is shown in Fig. 11. For more than one power output state, the state-space diagram becomes a diagram of multiple layers, where each layer represents a two-dimensional power output state of the WFs and all operating states of the WFVSC stations.

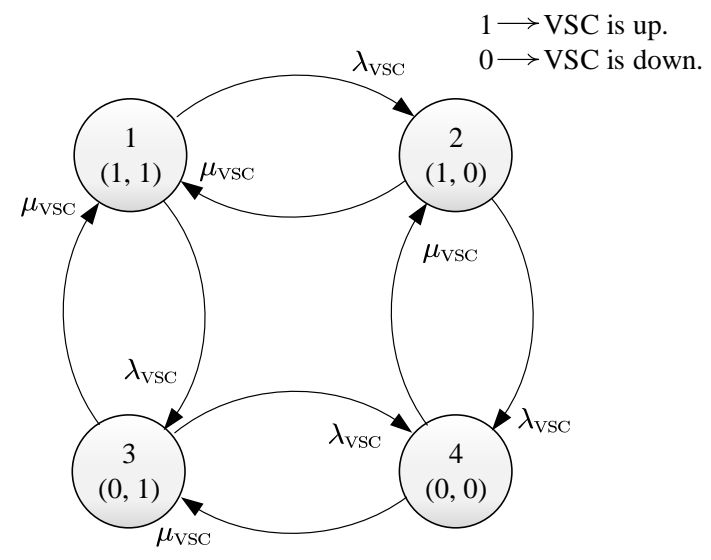

Fig. 11. State-space diagram of EM_1 for one power output state.

Due to the series structure of the GSVSC and DCTL, the series equivalent method can be used to obtain EM_2. It is a two-state Markov model.

Combining EM_1 and EM_2, the final combined reliability model can be obtained. For each state, the available generating capacity of the wind energy system is the sum of the two offshore WFs. The total number of states in the combined model is $20 \times 4 \times 2=160$, which is too large for reliability assessment. Therefore, the almost identical states are merged again. Here, the available generating capacity of the system is divided into 0-, 10-, 20-, 30- 40-, and 50- MW steps. Then, the combined model can be represented by a six-state Markov model as shown in Fig. 12. Table IV shows the capacity outage probability table (COPT) of the combined model where $\lambda_{+}$and $\lambda_{-}$denote the departure rates to the higher and lower generation capacity states, respectively.

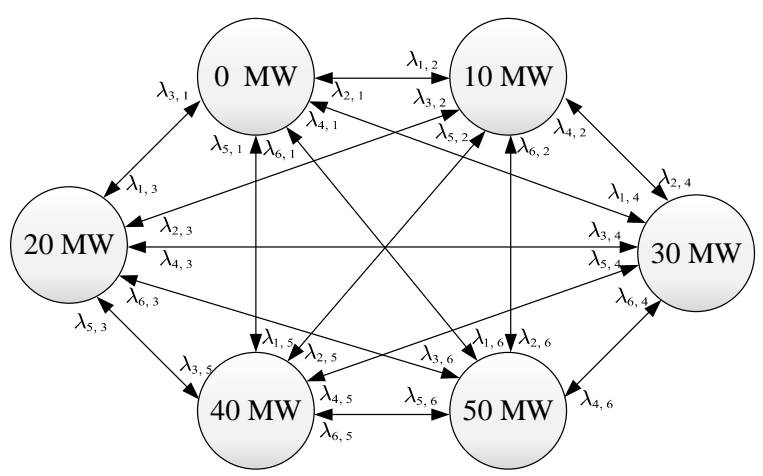

Fig. 12. Six-state Markov model of the VSC-HVDC connected offshore WFs.

TABLE IV

COPT OF THE COMBINED RELIABILTY MODEL

\begin{tabular}{c|c|c|c|c|c}
\hline \hline State & $\begin{array}{c}\text { Capacity in } \\
\text { (MW) }\end{array}$ & Probability & $\begin{array}{c}\lambda_{+} \\
\text {(occ/hr) }\end{array}$ & $\begin{array}{c}\lambda_{-} \\
\text {(occ/hr) }\end{array}$ & $\begin{array}{c}\text { Frequency } \\
\text { (occ/hr) }\end{array}$ \\
\hline \hline 1 & 0 & 0.066675 & 0.278099 & 0 & 0.018542 \\
\hline 2 & 10 & 0.420041 & 0.153089 & 0.068648 & 0.093139 \\
\hline 3 & 20 & 0.181092 & 0.300682 & 0.174809 & 0.086108 \\
\hline 4 & 30 & 0.115783 & 0.135387 & 0.210523 & 0.040051 \\
\hline 5 & 40 & 0.139796 & 0.159471 & 0.245926 & 0.056673 \\
\hline 6 & 50 & 0.076612 & 0 & 0.136466 & 0.010453 \\
\hline \hline
\end{tabular}

\section{Generation System Reliability Assessment With OFFSHORE WFS}

The combined multi-state model of VSC-HVDC connected offshore WFs can be treated as a conventional generation unit with derated power output states. In this paper, the basic reliability assessment technique is used to form the COPT of the entire generation system. If a state includes different states of conventional generating units and the offshore WFs, the probability, transition rates and frequency can be obtained using the following equations [6],

$$
\begin{gathered}
p_{s}=\prod p_{i} \\
\lambda_{ \pm s}=\sum \lambda_{ \pm i} \\
f_{s}=p_{s}\left(\lambda_{+s}+\lambda_{-s}\right)
\end{gathered}
$$

where $p, f, \lambda_{+}$and $\lambda_{-}$are the probability, frequency, and departure rates to the higher and lower generation capacity states, respectively, $s$ refers to the state resulting from the combination of different states of generating units, and $i$ represents the operating states of different generating units in the state $s$. Those identical states can be merged by,

$$
\begin{aligned}
p_{k} & =\sum p_{i} \\
f_{k} & =\sum f_{i} \\
\lambda_{ \pm k} & =\frac{\sum p_{i} \lambda_{ \pm i}}{p_{k}}
\end{aligned}
$$

where the subscript $i$ refers to the identical states, and $k$ 
refers to the merged state.

The hourly load values are grouped into $K$ class levels.

The mean of each class is taken as the load level value [21]. The frequency, probability and transition rates are calculated. Then, a margin model can be obtained by convolving the generation model and load model. The identical margin states can be merged using (10.a)-(10.c). The first negative margin state is taken as the loss of load situation.

\section{CASE STUDY}

In this section, the modified RBTS is used to verify the proposed model. The original RBTS has 11 conventional generating units with total $240 \mathrm{MW}$ installed capacity and 185 MW peak load [25]. The original load data are grouped into 20 levels. The reliability indices include loss of load expectation (LOLE), expected energy not supplied (EENS), loss of load frequency (LOLF) and loss of load duration (LOLD). To demonstrate the accuracy and efficiency of the proposed combined model, the simulation results using the proposed model are compared with the results using the analytical model, in which the wind speed correlation is modelled using the Copula method [13] and the conventional sequential MCS method, which is generally used for validation. The effects of the VSC-HVDC system and wind speed correlation on system reliability are analyzed. Moreover, sensitivity analyses were conducted to investigate the impact of the repair time of the offshore VSC-HVDC system on system reliability.

\section{A. Validation of the Combined Model}

In this study, the coefficient of variation for EENS, set as 0.01 , is used as the convergence criterion for the sequential MCS method. The Gaussian Copula function is adopted in the Copula method. Moreover, it should be mentioned that according to the method in [13], the proposed Copula method cannot be used to calculate the frequency indices LOLF and LOLD.

Fig. 13 shows the reliability assessment results with different peak load levels. As can be seen from Fig. 13, with different load levels, the probability and frequency indices using the combined model are close to those with the sequential MCS method, which demonstrates the effectiveness and accuracy of the proposed combined model. Additionally, the Copula method also shows effectiveness in reliability assessment, whereas the combined model shows better accuracy.

The computation time of reliability assessment using the combined model and the Copula method are less than 1s, whereas the computation time using the sequential MCS method is about 150s. Consequently, the combined model and the Copula method are more efficient for reliability assessment. Considering the complicated work for parameter estimation in the Copula method, the analytical model based on the clustering method is easier for implementation.

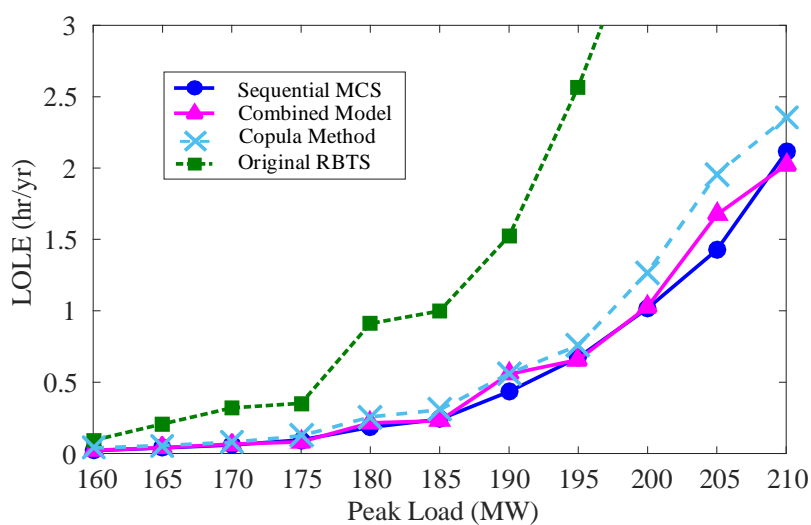

(a)

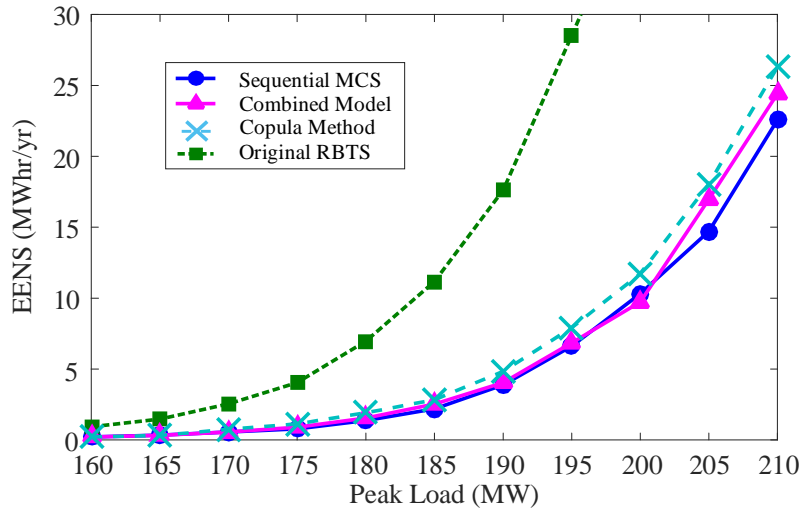

(b)

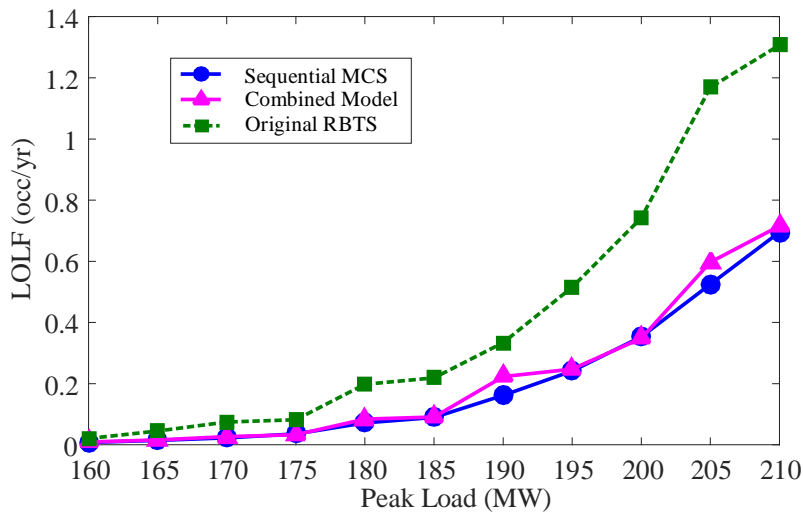

(c)

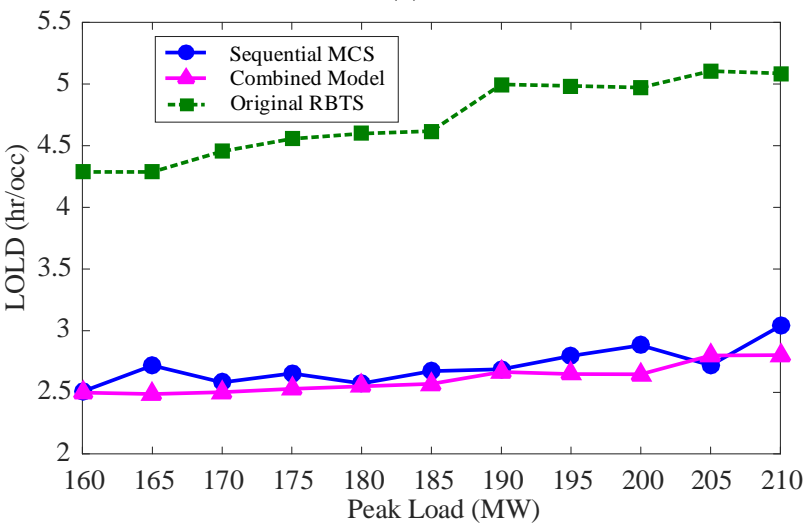

(d)

Fig. 13. Reliability indices with different peak load levels. (a) LOLE; (b) EENS; (c) LOLF; (d) LOLD. 


\section{B. Effects of VSC-HVDC System and Wind Speed Correlation}

To investigate the effects of wind speed correlation and outage of the VSC-HVDC system on system reliability, the following four cases are considered in this study.

Case 1: The original RBTS without wind power.

Case 2: The modified RBTS with offshore wind power considering wind speed correlation and the failures of the VSC-HVDC system (the proposed combined model).

Case 3: The modified RBTS with offshore wind power only considering wind speed correlation.

Case 4: The modified RBTS with offshore wind power only considering the outage of VSC-HVDC systems.

The reliability indices of the RBTS in the four cases are calculated and listed in Table V. Comparing Case 2 with Case 3 , it is shown that the outage of the VSC-HVDC slightly reduce the system reliability level. Neglecting the outage of the VSC-HVDC system will lead to an inaccurate overoptimistic assessment results. Comparing Case 2 with Case 4, it is shown that the over-optimistic assessment results are also obtained without considering wind speed correlation. In Case 4, the WFs are modeled independently using the single WF model [6]-[7]. Hence, the correlation between two WFs is zero. Since lower correlation between WFs leads to stronger complementarity, the risk indices are reduced.

TABLE V

RELIABILITY INDICES OF DIFFERENT CASES

\begin{tabular}{c|c|c|c|c}
\hline \hline Case & $\begin{array}{c}\text { LOLE } \\
(\mathrm{hr} / \mathrm{yr})\end{array}$ & $\begin{array}{c}\text { EENS } \\
(\mathrm{MWhr} / \mathrm{yr})\end{array}$ & $\begin{array}{c}\text { LOLF } \\
\text { (occ/yr) }\end{array}$ & $\begin{array}{c}\text { LOLD } \\
\text { (hr/occ) }\end{array}$ \\
\hline 1 & 0.9988 & 11.1710 & 0.2192 & 4.5564 \\
\hline 2 & 0.2297 & 2.5234 & 0.0909 & 2.5286 \\
\hline 3 & 0.2241 & 2.4605 & 0.0885 & 2.5336 \\
\hline 4 & 0.1656 & 1.8189 & 0.0667 & 2.4810 \\
\hline \hline
\end{tabular}

\section{Effect of the Repair Time of Offshore VSC-HVDC System}

Due to lack of data of offshore WFs and VSC-HVDC systems, the outage data used in the modeling are obtained from onshore HVDC systems. However, the failure rates and repair time of the offshore VSC-HVDC system are quite different from the onshore cases [17]. For example, the repair work significantly depends on the weather conditions such as wind regime and wave height. The resulting repair time may be much longer than that of onshore cases.

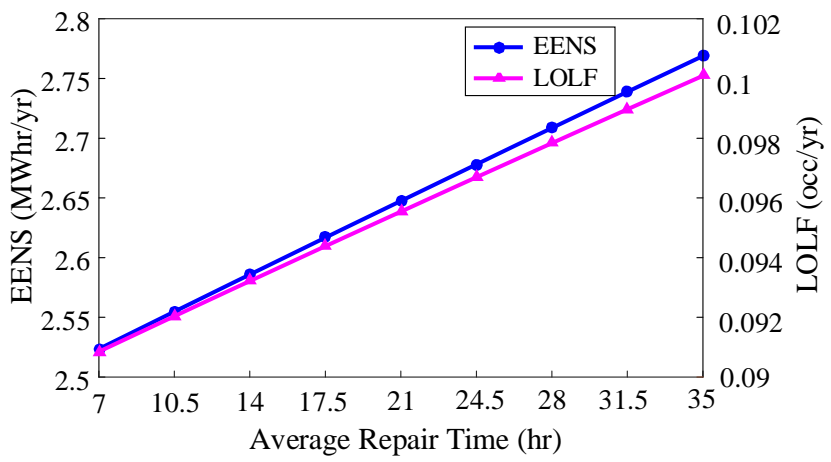

Fig. 14. EENS and LOLF with different average repair time.
Sensitivity analyses are analyzed with different repair time. Fig. 14 shows the EENS and LOLF with different average repair time of WFVSC stations. Obviously, as the average repair time increases, the EENS and LOLF both increase. And an approximately linear relationship can be observed.

\section{CONCLUSION}

This paper proposes a combined reliability model of VSCHVDC connected offshore WFs considering wind speed correlation. The global fast k-means clustering method is used to model the correlation between WFs. The reliability model of a multi-terminal VSC-HVDC system is developed and then combined with the WF model.

The effectiveness and accuracy of the proposed model are validated by comparing simulation results with sequential MCS method. The comparison shows that the reliability assessment results with the proposed model are almost identical to the ones with the MCS methods, which verifies the accuracy of the proposed model. Moreover, the proposed model shows better computation efficiency than the MCS method in reliability assessment.

The outage of the VSC-HVDC system and wind speed correlation can both affect the system reliability. Neglecting them in reliability assessment will lead to over-optimistic assessment results. Sensitivity analyses show the effect of the repair time of the offshore VSC-HVDC system on system reliability. With the increase of repair time, the EENS and LOLF indices both increase and have an approximately linear relationship with the repair time.

\section{APPENDIX}

Table VI and VII show the original outage data of DFIGbased WTG and VSC-HVDC system used in the model.

TABLE VI

WTG OUTAGE DATA

\begin{tabular}{c|c|c|c}
\hline \hline Components & $\begin{array}{c}\text { Failure rate } \\
\text { (occ/yr) }\end{array}$ & $\begin{array}{c}\text { Average repair } \\
\text { time (hr) }\end{array}$ & Unavailability \\
\hline \hline Tower & 0.006 & 104.1 & $7.1301 \mathrm{E}-5$ \\
\hline Hub & 0.001 & 12.5 & $1.4269 \mathrm{E}-6$ \\
\hline Blades/Pitch & 0.052 & 91.6 & 0.000543 \\
\hline Gearbox & 0.045 & 256.7 & 0.001317 \\
\hline Drive train & 0.004 & 291.4 & 0.000133 \\
\hline Generator & 0.021 & 210.7 & 0.000505 \\
\hline Yaw & 0.026 & 259.4 & 0.000769 \\
\hline Sensors & 0.054 & 49.4 & 0.000304 \\
\hline Control system & 0.050 & 184.6 & 0.001053 \\
\hline Brake system & 0.005 & 125.4 & $7.158 \mathrm{E}-5$ \\
\hline Converters & 0.067 & 106.6 & 0.000815 \\
\hline Transformer & 0.020 & 200 & 0.000456 \\
\hline \hline
\end{tabular}

TABLE VII

VSC-HVDC SYSTEM OUTAGE DATA

\begin{tabular}{c|c|c|c}
\hline \hline Components & $\begin{array}{c}\text { Failure rate } \\
\text { (occ/yr) }\end{array}$ & $\begin{array}{c}\text { Average repair } \\
\text { time (hr) }\end{array}$ & Unavailability \\
\hline \hline Valves & 0.5 & 4 & $2.28311 \mathrm{E}-5$ \\
\hline CR & 0.006585 & 25 & $1.87928 \mathrm{E}-5$ \\
\hline Trn & 0.0309 & 24 & $8.46575 \mathrm{E}-5$ \\
\hline Cap & 0.05 & 11.55 & $6.59247 \mathrm{E}-5$ \\
\hline \multicolumn{3}{|c}{}
\end{tabular}




\begin{tabular}{c|c|c|c}
\hline ACF & 0.0833 & 10.5 & $9.98459 \mathrm{E}-5$ \\
\hline DCF & 0.4 & 12 & $5.47945 \mathrm{E}-4$ \\
\hline PCS & 1.3095 & 8 & 0.00119589 \\
\hline Brk & 0.001 & 40 & $4.56621 \mathrm{E}-6$ \\
\hline DCTL $(/ \mathrm{km})$ & $3 \mathrm{E}-5$ & 5 & $1.71233 \mathrm{E}-8$ \\
\hline \hline
\end{tabular}

\section{REFERENCES}

[1] Wind energy scenarios for 2030, European Wind Energy Association. [Online].Available:https://windeurope.org/fileadmin/files/library/publica tions/reports/EWEA-Wind-energy-scenarios-2030.pdf.

[2] L. Xu, L. Yao, and C. Sasse, "Grid integration of large DFIG-based wind farms using VSC transmission,” IEEE Trans. Power Syst., vol. 22, no. 3, pp. 976-984, Aug. 2007.

[3] Y. Ding, C. Singh, L. Goel, J. Østergaard, and P. Wang, "Short-term and medium-term reliability evaluation for power systems with high penetration of wind power," IEEE Trans. Sustain. Energy, vol. 5, no. 3, pp. 896-906, Jul. 2014.

[4] R. Billinton and Y. Gao, "Multistate wind energy conversion system models for adequacy assessment of generating systems incorporating wind energy,” IEEE Trans. Energy Convers., vol. 23, no.1, pp. 163-170, Mar. 2008.

[5] A. P. Leite, C. L. T. Borges, and D. M. Falcão, "Probabilistic wind farms generation model for reliability studies applied to Brazilian sites,” IEEE Trans. Power Syst., vol. 21, no. 4, pp. 1493-1501, Nov. 2006.

[6] A. S. Dobakhshari and M. Fotuhi-Firuzabad, "A reliability model of large wind farms for power system adequacy studies," IEEE Trans. Energy Convers., vol. 24, no.3, pp. 792-801, Sep. 2009.

[7] A. Ghaedi, A. Abbaspour, M. Fotuhi-Firuzabad, and M. Moeini-Aghtaie, "Toward a comprehensive model of large-scale DFIG-based wind farms in adequacy assessment of power systems," IEEE Trans. Sustain. Energy, vol. 5, no. 1, pp. 55-63, Jan. 2014.

[8] W. Wangdee and R. Billiton, "Considering load-carrying capability and wind speed correlation of WECS in generation adequacy assessment," IEEE Trans. Energy Convers., vol. 21, no.3, pp. 734-741, Sep. 2006.

[9] R. Billinton, Y. Gao, and R. Karki, "Composite system adequacy assessment incorporating large-scale wind energy conversion systems considering wind speed correlation,” IEEE Trans. Power Syst., vol. 24, no. 3, pp. 1375-1382, Nov. 2009.

[10] R. Billiton, P. Karki, Y. Gao, D. Huang, P. Hu, and W. Wangdee, "Adequacy assessment considerations in wind integrated power systems," IEEE Trans. Power Syst., vol. 27, no. 4, pp. 2297-2305, Nov. 2012.

[11] S. Wang, X. Zhang, L. Ge, and L. Wu, "2-D wind speed statistical model for reliability assessment of microgrid,” IEEE Trans. Sustain. Energy, vol. 7, no. 3, pp. 1159-1169, Jul. 2016.

[12] M. Mosadeghy, R. Yan, and T. K. Saha, "A time-dependent approach to evaluate capacity value of wind and solar PV generation,” IEEE Trans. Sustain. Energy, vol. 7, no. 1, pp. 129-138, Jan. 2016.

[13] C. Fan, F. Li, Z. Wei, G. Sun, and J. Li, "Reliability models of wind farms considering wind speed correlation and WTG outage," Electr. Power Syst. Res., vol. 119, pp. 385-392, 2015.

[14] L. He, C. Liu, A. Pitto, and D.Cirio, "Distance protection of AC grid with HVDC-connected offshore wind generators," IEEE Trans. Power Del., vol. 29, no. 2, pp. 493-501, Apr. 2014.

[15] J. N. Sakamuri, Z. H. Rather, J. Rime, M. Altin, O. Goksu, and N. A. Cutululis, "Coordinated voltage control in offshore HVDC connected cluster of wind power plants,” IEEE Trans. Sustain. Energy, vol. 7, no. 4, pp. 1592-1601, Oct. 2016.

[16] S. Zadkhast, M. Fotuhi-Firuzabad, F. Aminifar, R. Billiton, S. O. Faried, and A. Edris, "Reliability evaluation of an HVDC transmission system tapped by a VSC station,” IEEE Trans. Power Del., vol. 25, no. 3, pp. 1962-1970, Jul. 2010.

[17] C. MacIver, K. R. W. Bell, and D. P. Nedić, "A Reliability evaluation of offshore HVDC grid configuration options,” IEEE Trans. Power Del., vol. 31, no. 2, pp. 810-819, Apr. 2016.

[18] B. Wang, X. Wang, X. Wang, C. Shao, and S. Liu, "Reliability evaluation of voltage source converter-based multi-terminal direct current integrated offshore wind plants,” IET Renew. Power Gener., vol. 10, no. 6, pp. 761-766, 2016.

[19] R. Billinton and R. Allan, Reliability Evaluation of Engineering Systems, 2nd ed. New York: Plenum, 1992.

[20] A. Likasa, N. Vlassisb, and J. Verbeekb, “The global k-means clustering algorithm,” Pattern recognition, vol. 36, no. 2, pp. 451-461, 2003.
[21] R. Billiton and R, Allan, Reliability Evaluation of Power Systems, 2nd ed. New York: Plenum, 1996.

[22] J. Ribrant and M. Bertling, "Survey of failures in wind power systems with focus on Swedish wind power plants during 1997-2005," IEEE Trans. Energy Convers., vol. 22, no.1, pp. 167-173, Mar. 2007.

[23] A. Korompili, Q. Wu, and H. Zhao. "Review of VSC-HVDC connection for offshore wind power integration," Renewable and Sustainable Energy Reviews, pp.1405-1414, 2016.

[24] M. Ding, R. Bi, and J. Wang, "FD method and combined model for reliability assessment of HVDC flexible,” Power Syst. Protect. Control, vol. 26, no. 21, pp. 33-37, Nov. 2008.

[25] R. Billiton, S. Kumar, N. Chowdhury, K. Chu, K. Debnath, L. Goel, E. Khan, P. Kos, G. Nourbakhsh and J. Oteng-Adjei, "A reliability test system for educational purposes basic data,” IEEE Trans. Power Syst., vol. 4, no. 4, pp.1238-1244, Aug. 1989. 